\title{
EE-MAC: Energy Efficient-Medium Access Control for Periodic Applications in Border Surveillance Wireless Sensor Networks
}

\author{
Sam Nguyen-Xuan, Semin Oh, and An Sunshin \\ School of Electrical Engineering, Korea University Seoul, Republic of Korea \\ \{ samnx,smoh,sunshin\}@dsys.korea.ac.kr
}

\begin{abstract}
This paper propose the energy saving algorithms for medium access control in wireless sensor network to improve energy saving and increasing packet delivery ration for periodic applications in border surveillance wireless sensor networks. Since the data dissemination is periodic, the data update can be scheduled so that all nodes in virtual cluster must tightly synchronize their transmission to avoid collision. S-MAC [1] and D-MAC [2] schedules should be modified to satisfy the power cost of data delivery. Our implementations of this paper proposed the wake-up algorithm, the delay wakeup algorithm, and topology based the virtual group schedules. The results show that our proposed algorithms perform better than S-MAC and D-MAC in terms of energy efficiency and packet delivery ratio.
\end{abstract}

Keywords - medium access control (MAC), border surveillance wireless sensor networks (BSWSNs), energy efficiency (EE), and packet delivery ratio (PDR).

\section{INTRODUCTION}

Wireless sensor networks (WSNs) have a wide range of potential applications due to inexpensive and small characteristics. Therefore, they are being developed to interact directly with the physical world. Commonly, sensors nodes can be deployed in specific conditions in predefine areas such as border, private properties. Their main objective is the detection able to sense various environmental data such as temperature, light, humidity, and vibration, node mobility, etc. there are huge border surveillance applications for WSNs, However, depend on application-specific goals, there are difference protocols that focus on the characteristics of application to optimal performance of WSNs. Our works consider battery lifetime and packet delivery ratio (PDR) in underwater surveillance wireless sensor network (USWSNs).

A large number of energy-efficient MAC protocols have been proposed, each protocol showed that it plays a most crucial rule in communication energy efficiency, based on the applications, each with each own specific trade-off. S-MAC [1] differs from other traditional wireless MAC protocols. In order to minimize the idle listening, S-MAC use a sleep-listen schedule, when slept, nodes turn off their radio. Nodes also synchronize each other by broadcasting synchronization (SYNC) packets and each node could follow more than one schedule. In this way, nodes can save their energy. This protocol trades off among energy efficiency, latency and throughput. D-MAC [2] is designed to solve the interruption problem by giving the active/sleep schedule of a node offset that depends upon the tree. D-MAC is to archive low latency and saving energy and support for more than two hops path. D-MAC also adjusts the node duty cycles adaptively according to the traffic load in the network. T-MAC [4] is proposed to enhance the performance result of S-MAC protocol under variable traffic load. In T-MAC, a node will keep listening and potential transmitting as long as it is in an active period, an active period ends when no activation event has occurred. T-MAC is efficient with high load. PAMAS [5], use an extra radio, called wake up radio, which operates on a different frequency than the radio used for communication. As the wake up radio is only walking up other nodes, no data processing and therefore use much less energy. Recently, asynchronous duty-cycle (DC) MAC such as SEA-MAC [3] B-MAC [7], X-MAC [8], and R-MAC [9] allow nodes to operate independently, with each node on its own duty cycle schedule. SEA-MAC [3] use signal packets to wake up nodes and move the packet through the networks if the nodes know period time of packets, in this way, they can turn off their radio. They achieve high energy efficiency and remove the synchronization overhead required in synchronous duty cycle approaches. However, asynchronous DC approaches become less efficient in latency and packet delivery ratio (PDR).

The different MAC protocols can be efficient depending on the given environment and their applications. Our works focus on managing a recreational beach area, thus the measurements will be collected every several minutes [6]. The environment applications include monitoring of physical indicators and chemical/biological indicators. Ones of the major considerations for this system are the power consumption and PDR. In order to improve battery lifetime and increase PDR for these systems, we consider the packet delivery, the distance, and the synchronization schedules impact on energy consumption. This work is driven by the need to develop a medium-specific aspects and topology structure aspects in wireless sensor network.

In this paper, firstly, we propose energy efficient algorithm deployed for our applications. In our scheme, we prolong lifetime for overall network based on the fact that the packet periods depends on periodic applications.

Secondly, virtual synchronization in S-MAC [1] will be reorganized to adapt with packet period characteristics. The 
number of nodes in a cluster has to reorganization their schedules into the sectors. The topologies perfectly adapt with the real environments.

Thirdly, the design of topology architecture is extend to support increasing PDR and improving energy efficiency. The distance-based topology and schedule-based topology reduce not only packet loss but also increase the higher degree of fairness and balance.

The remainder of this paper is organized as follows: Section 2 includes challenges of WSWSNs. Section 3 describes our approaches. In section 4, we evaluates the performance analyse, and section 5 is our conclusions and future works.

\section{Challenges in USWSNS}

The development of the MAC protocol for USWSNs is challenging due to energy limitation of nodes, low data rate, long propagation latency, and synchronization. Minimizing energy consumption while maximizing PDR require networks less frequency updates, multi-hop and shorter communication range.

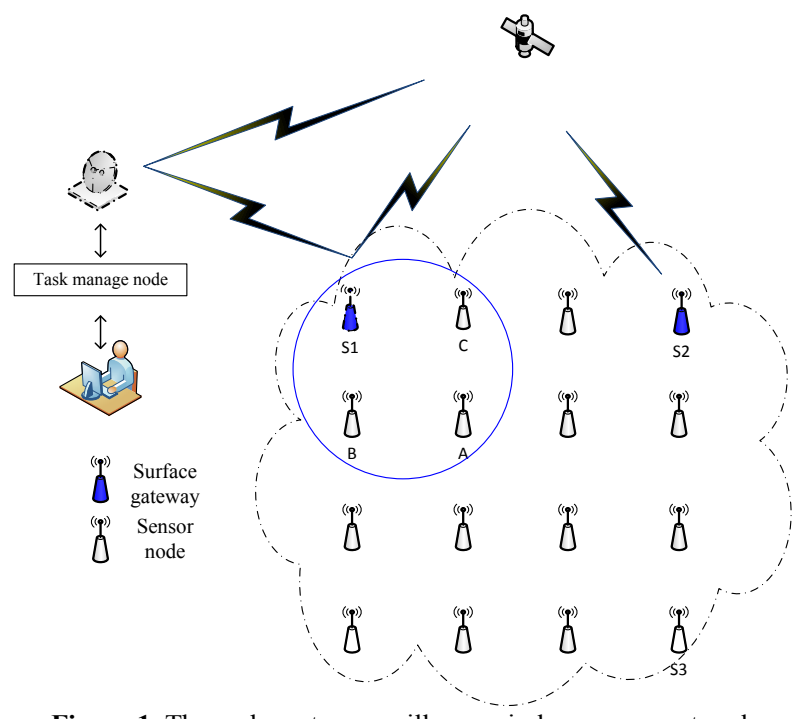

Figure 1. The underwater surveillance wireless sensor network

The USWSN senses environment state and determine risk level of environment is described in figure 1. In this figure, nodes monitor their surrounding environment conditions and periodically send the collected information to toward to surface gateway.

Basically, energy efficiency that addresses these challenges affects both energy lifetime of whole network and PDR. There are four main states which energy consumption for transmitting $\left(E_{t x}\right)$, energy consumption for receiving $\left(E_{r x}\right)$, energy consumption for listening $\left(E_{\text {listen }}\right)$ and energy consumption for sleeping $\left(E_{\text {sleep }}\right)$ effect on energy consumption of each node. Therefore, energy is consumed by each node following below

$$
E=E_{t x}+E_{r x}+E_{\text {listen }}+E_{\text {sleep }}
$$

In case of energy consumption for transmitting and receiving, energy model can be applied from [10] to calculate energy consumption. Therefore, the energy consumed when the sensors receive a packet of size $k$ is given:

$$
E_{r x}=E_{\text {elec }} \times k
$$

Energy consumed by sending a packet of size k:

$$
E_{t x}=E_{\text {elec }} \times k+E_{\text {amp }} \times k \times r^{2}
$$

where $E_{\text {elec }}$ is the energy that the radio dissipates to run the circuitry for the transmitter and receiver, $E_{a m p}$ is the energy for the transmit amplifier. From (2) and (3), $E_{t x}>>E_{r x}$ when distance between sensor nodes and size of a packet is increased.

In case of energy consumption for listening and sleeping, there are major sources of energy wastage are identified in and described below:

- Idle listening: listening to an idle channel in order to receive possible traffic.

- Collision: Discarded and Retransmission

- Overhearing

- Control Packet Overhead

- Over-emitting: the transmission of a message when the destination node is not ready

Since the data dissemination is periodic, the data update can be scheduled so that all nodes in virtual cluster must tightly synchronize their transmission to avoid collision. SMAC and D-MAC schedules should be modified to satisfy the power cost of data delivery. We consider data period based on message inter arrival and data sampling to change listening/sleeping S-MAC and D-MAC schedules which effect on distance latency, size of packets, update period.

USWSNs communications are constrained by several factors that challenge their use in real environments. When the distance between the transmitter and the receiver increases, the receiving signal levels decrease at the receivers. This phenomenon is called path loss. Attenuation of radio signals has been modelled by averaging the measured signal powers over long times and over various locations with the same distances to the transmitter. The path loss $(\mathrm{dB})$ between the transmitting and the receiving antennas is a function of the distance $r$ :

$$
P L(d b)=P L_{0}+10 \eta \log \left(\frac{r}{r_{0}}\right)
$$

where $P L_{0}$ is path loss at distance $r_{0}, \eta$ is the path-loss exponent. The equation (4) applies for free space in wireless sensor networks but it does not capture the specific conditions of USWSN in which the path loss depend on frequency and distance. Moreover, propagation delay and multipath effects on the USWSNs are different with free space environments. As reported in [11], authors provide the following equation for transmission loss:

$$
P L(d b)=10 \log r+\alpha r \times 10^{3}
$$

where $\alpha$ is the frequency dependent medium absorption coefficient. This equation show that the nodes transmit their packets at higher frequency $(\mathrm{KHz})$, they spend more energy consumption. Therefore, the transition power depends on the distance and frequency. This paper we consider only the distance dependent and update period which impact on energy consumption of nodes.

In order to avoid sending packets over long distance and large energy consumption, the tight schedules are assigned for 
the sensor nodes in the hierarchical topology and the cluster self-organization is considered.

\section{DESCRIPTION OF OUR APPROACHES}

In our applications, we focused on S-MAC [1] and D-MAC [2] since it is suitable for periodic applications. However, in order to avoid much consuming power for sending/receiving packets overlong distance and low and period traffic, we really need to improve these protocols. This work is motivates by practical need to estimate the battery lifetime of wireless sensor nodes, which has implications on the usefulness, topology and range of the network. The fundamental tasks of this implementation have to ensure it works well in light and period traffic, energy efficiency, PDR, and require strictly latency.

In S-MAC, if periods to transmit data are larger than a large number of frames, in this situation, the nodes no need to wake up in the next periods, and therefore, more energy would be saved because the nodes sleep. The modifying SYNC depended on above application's periods is necessary to save more energy. We compared our proposal with S-MAC which is described in figure 2 . In our proposal, the listening periods between two sampled packets of the nodes should be turned their radio off. They can flexible their schedules depend on the period of each applications.

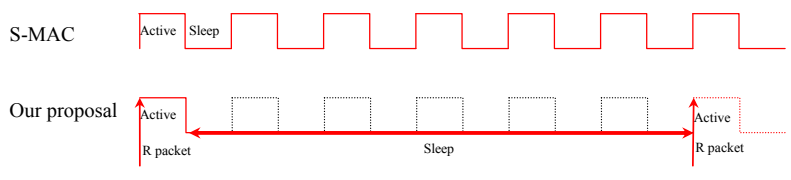

Figure 2. The sampling wake-up scheme

We value energy consumption for MAC protocol where a node accesses the channel, sends a data packet, and awaits an acknowledgment. Energy consumption of node $i^{\text {th }}$ at MAC is following:

$$
\begin{aligned}
& E_{\text {listen }}=\left(\frac{1}{T_{f}}-2 \times n(i)\right) T_{0} \times E_{\text {listen }}(i) \\
& E_{\text {sleep }}=\frac{T_{0}}{T_{f}} \times E_{\text {sleep }}(i)
\end{aligned}
$$

where $l$ is time between two sample packets, $T_{0}$ is sample period, $n(i.) T_{0}$ is number of packet received or transmitted by node $\mathrm{i}^{\text {th }}, T_{f}$ is the period time of frame in S-MAC and DMAC. Suppose that the time between two sample packets is very larger than the period time of frame.

There are two appeared problems when we modified SYNC depend on period of applications. First of all, the problem comes from virtual group synchronization which the nodes closer to the sink may work with multiple schedules and the second problem which impact on SYNC skew. In the first situation, the nodes may exhaust their energy due to multiple schedules while the second problem usually happens with the USWSNs, whenever the packets are sent over long distance and the conditions of underwater medium are changed frequently, both of these problems make the nodes, especially the nodes closer to the gateway, will pay cost for their miss wakeup (early wakeup), higher packet dropt and more energy consumption which spend for retransmission and idle listening of the nodes are increased.

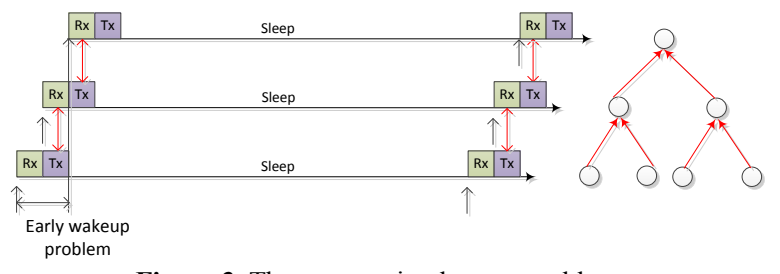

Figure 3. The propagation latency problem

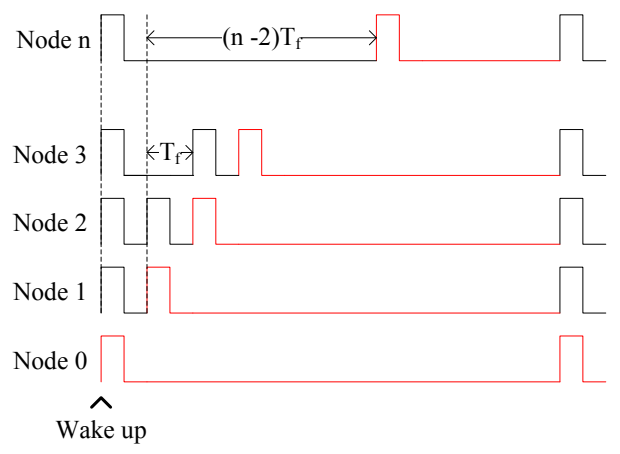

Figure 4. The delay wakeup algorithms

Our optimal schedules from S-MAC based on hierarchical topologies and multipath routing which the nodes can forward their packets to the surface gateway in parallel. First of all, the nodes closer to the gateway in a virtual cluster could divide in a sector, multi-sectors in cluster work parallel. If the nodes closer to the sink maintained the more than two schedules in a virtual group, they follow the first schedule and request help from the nodes which are the same level, and then they check their own sequence number to back off their schedule. We show the delay wakeup scheme in figure 4. This algorithm allows node closer to the gateway surface to delay wake-up in from one fame period to $n$ frame period, this depend on the hop sequence number record from routing phase.

Since multi-path routings are used, the multi schedules are divide to single schedule, this create a virtual cluster into parallel chains, then, the topologies should change from chain topology or tree topology to grip topology. A grip topology needs extension to support for packet delivery and energy efficiency, this approach shows that the failure of any forwarded nodes does not cause the system failure. Furthermore, if the energy consumption spends for transmitting and receiving of a packet that is different such as reported [12], the receive energy of each packet is around one fifth of transmit energy. The nodes have to pay more energy cost whenever the probability of the transmitting packets and the probability of receiving packets are lower than one. For improving this point, we then present these relationships as network lifetime functions. The example of these relationships is described in figure 1 (blue circle) in which $R_{A}$, $R_{B}$, and $R_{C}$ are remain energy of nodes $A, B$, and $C$, the lifetime of this grid lattice is a function as follows:

$$
R=1-\left(1-R_{A}\right)\left(1-R_{B} \times R_{C}\right)
$$


The hierarchical grid-based formation in this example shows that the depletion of any forwarded nodes will not cause the block failure while in traditional architecture such as the chain topology or tree topology, the failure of any parent nodes will cause the system failure. Therefore, the grid based on the rectangular grid can improve reliability for forwarding packets. On the other hand, the members in this virtual group can create schedules two chains $\left(\mathrm{ABS}_{1}\right.$ and $\left.\mathrm{ACS}_{1}\right)$, this work makes not only flexible scheduler in virtual group but also increase reliability for forwarding packets.

Energy efficient (EE)-MAC combine both solutions of the exchange SYNC problem and the propagation latency problem. We optimal our proposals on grid-based topology which can flexible schedules of the nodes in a virtual cluster which can create the parallel forwarding chains.

\section{IV.PERFORMANCE ANALYSIS}

In order to evaluate performance of our algorithms, we perform several simulations in ns-2 [14] simulation. sixteen nodes are deployed in $1000 \times 1000 \mathrm{~m}^{2}$ in which the initial energy of each node is set to $10(\mathrm{~kJ})$. Each round, nodes send their packets with 100 (bytes) and there are parallel sources have been sent their packets. We also vary the values of some factors to determine probability of successful transmission for this payload size. In figure 5, the grid topology is formed, the AOMDV routing protocol [13] in cooperated in the network layer and this topology can support various kinds of application such as constant bit rate (CBR). The parameters used in the simulations are given in table 1 . Table 1 is given symbols and parameters used in modified and analyzed in our proposal. The simulations were run for 10000 seconds. We selected the duty cycles (DC) which are small (below 10\% DC) suitable with characteristics of our applications.

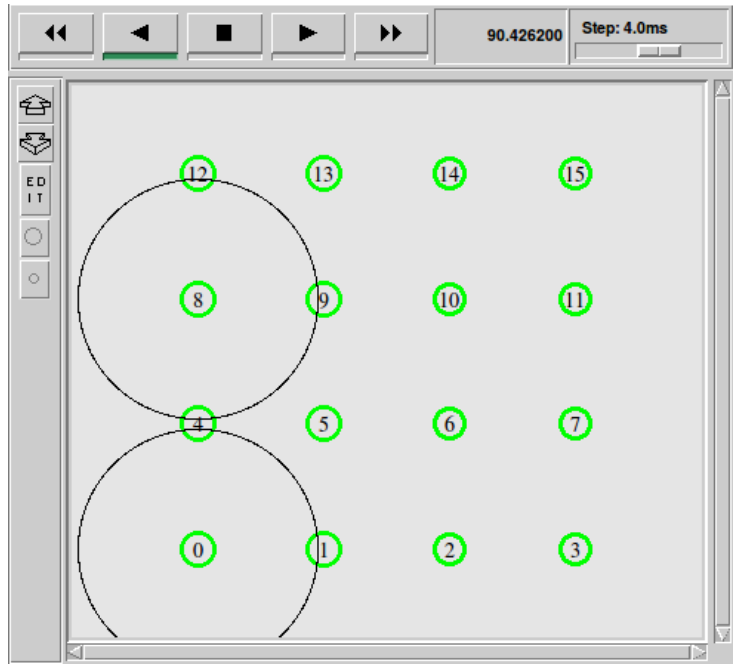

Figure 5. The grip $4 \times 4$ used in the simulation
TABLE 1: SIMULATED PARAMETERS

\begin{tabular}{|l|l|}
\hline parameters & Value \\
\hline $\mathrm{E}_{\mathrm{tx}}$ & $1.0 \mathrm{~mJ}$ \\
$\mathrm{E}_{\mathrm{rx}}$ & $0.2 \mathrm{~mJ}$ \\
$\mathrm{E}_{\mathrm{idle}}$ & $1.0 \mathrm{~mJ}$ \\
$\mathrm{E}_{\text {sleep }}$ & $0.001 \mathrm{~mJ}$ \\
$\mathrm{~T}_{0}$ & Varying \\
SYNC period & Varying \\
Packet rate & Varying \\
Length of CBR packets & 100 byte \\
Length of signal packets & 3 byte \\
Number of nodes & 16 \\
\hline
\end{tabular}

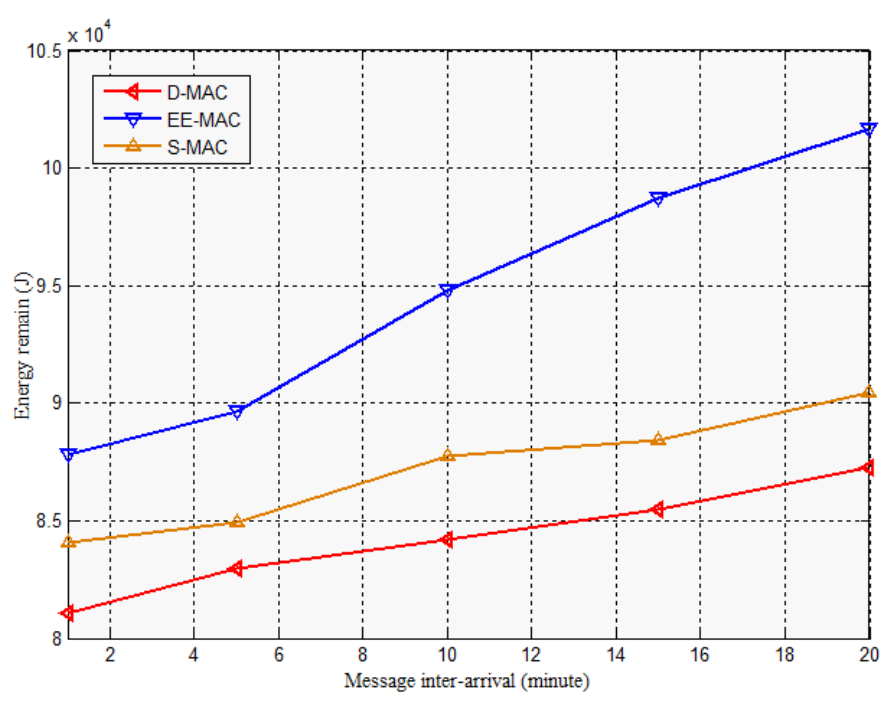

Figure 6. The energy remain

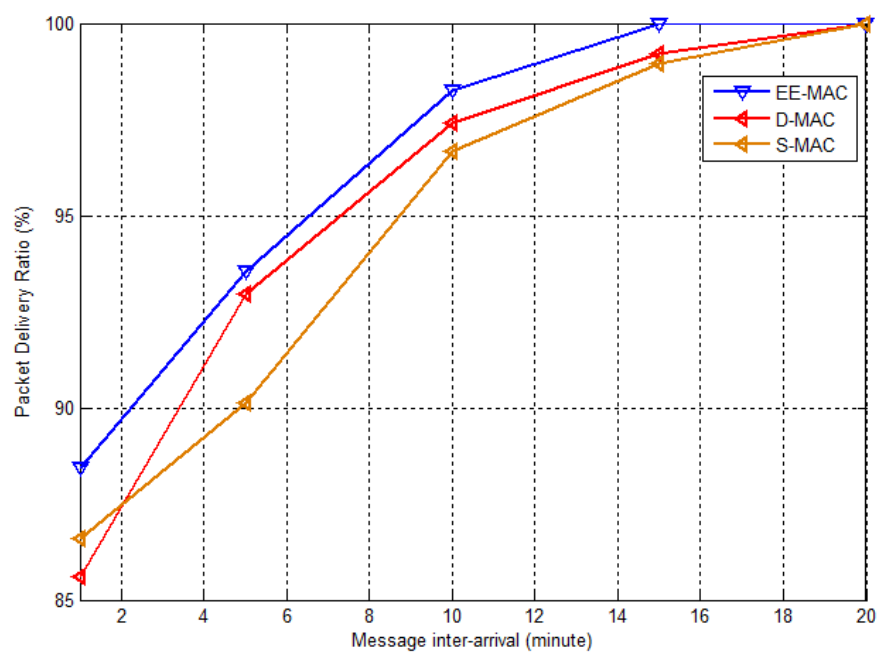

Figure 7. The packet delivery ratio

Figure 6 shows the remained energy in these simulations, the performance of EE-MAC is better than S-MAC and DMAC. It saves $23.08 \%$ compared with S-MAC and $32.25 \%$ compared with D-MAC.

Figure 7 shows the result of the packet delivery ratio for EE-MAC with varying number of active sources up to 2 , the packet delivery ratio of EE-MAC is better, however, it look slowly changes compared with S-MAC and D-MAC. This 
means that the trade-off between energy consumption and reliability affected PDR, the lower dropping of packets, the higher energy consumption.

\section{Conclusions}

This paper, firstly identify energy inefficient problem in MAC that results from the general synchronization policies for all sensor node in a virtual group. We then propose the turn off radio to extend the lifetime of nodes.

Secondly, we propose a simple but efficient architecture by extending chain topology or tree topology to the grid topology which makes flexible, reliable, efficient energy balance for packet delivery. Our schemes take into account not only propagation latency problem but also SYNC skew problem which usually happened in real environments.

Thirdly, the optimal topology is extended to support increasing PDR and improving energy efficiency. The distance-based topology and schedule-based topology reduce not only packet loss but also increase the higher degree of fairness and balance.

We prove that the impact on energy consumption related with probability of receiving and transmitting for a packet. The increasing above probability can extend the battery lifetime of nodes, thus, topology based and SYNC based are important aspects to improve this parameter.

\section{ACKNOWLEDGMENT}

This work was supported partially by the National Research Foundation of Korea (NRF) grant funded by the Korea government (MEST) (No. NRF-2012K1A3A1A09026959).

\section{REFERENCES}

[1] Wei. Ye, John. Heidemann and D.Estrin, "Medium access control with coordinated adaptive sleeping for wireless sensor networks", IEEE/ ACM Transactions on Networking, VOL. 12, NO 3, June 2004

[2] Gang Lu, Bhaskar Krishnamachari, Cauligi S. Raghavendra, "An adaptive energy-efficient and low-latency MAC for data gathering in sensor networks", in ProceedingsofIPDPS'2004, April 2004

[3] Miguel A. Erazo, Yi Qian, "SEA-MAC: A Simple Energy Aware MAC Protocol for Wireless Sensor Networks for Environmental Monitoring Applications," ISWPC '07.

[4] T. van Dam and K. Lanbendoen, "An adaptive energy-efficient MAC protocol for wireless sensor networks," in Proc. ACM Sensys 2003 Nov. 2003, pp. 171-180.

[5] S. Singh and C. S. Raghavendra, "PAMAS: Power aware multi-access protocol with signaling for ad hoc networks," ACM Comput. Commun. Rev., VOL. 28, NO 3, July 1998.

[6] Ian F. Akyildiz *, Dario Pompili, Tommaso Melodia, "Underwater acoustic sensor networks: research challenges", Ad Hoc Networks, 2005 .

[7] J. Polastre, J. Hill, and D. Culler, "Versatile Low Power Media Access for Wireless Sensor Networks", SenSys 2004.

[8] Michael Buettner, Gary V. Yee, Eric Anderson, Richard Han, "X-MAC: A Short Preamble MAC Protocol for Duty-Cycled Wireless Sensor Networks", SenSys 2004.
[9] Yanjun Sun, Omer Gurewitz, and David B. Johnson, "A Receiver Initiated Asynchronous Duty Cycle MAC Protocol for Dynamic Traffic Load", SenSys 2008.

[10] Wendi Rabiner Heinzelman, Anantha Chandrakasan, and Har Balakrishnan, "Energy-Efficient Communication Protocol for Wireless Micro sensor Networks", Proceedings of the 33rd Annual Hawaii International Conference on System Sciences, 2000.

[11] R. J. Urick, Principles of underwater sound, NewYork: McGraw Hill, 1983.

[12] "Underwater acoustic modem," www.link-quest.com.

[13] Mahesh K. Marina, Samir R. Das, "Ad hoc on-demand multipath distance vector routing", Wireless Communications and Mobile Computing, 2006

[14] The Network Simulator - ns-2, http://www.isi.edu/nsnam/ns/

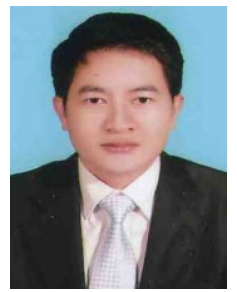

Sam Nguyen-Xuan received the B.S. degree in Electronics and Telecommunications Engineering from Posts and Telecommunications Institute of Technology (PTIT), Hanoi, Vietnam in 2002, and the M.S. degree in Information and Communications Engineering from the Andong National University, Republic of Korea, in 2009 $\mathrm{He}$ is currently pursuing the $\mathrm{Ph} . \mathrm{D}$. degree in Electrical and Computer Engineering at Korea University, Republic of Korea. His research interests include information theory, wireless sensor networks and ad-hoc networks.

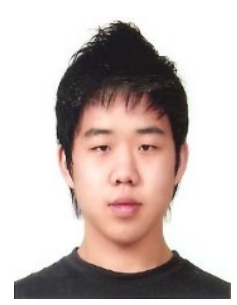

Semin Oh received the B.S. degree in Electronic Engineering from Sangmyung University, Korea, in 2012. Currently, he is pursuing for M.Sc. degree in Electronic and Computer Engineering at Korea University, Korea. His research interests include the information theory and wireless sensor networks.

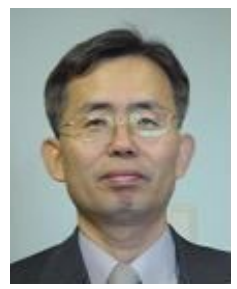

Sunshin An was born in Seoul, Korea in 1950. He received the B.Eng degree from Seoul National University, Korea in 1973, and the M.S degree in electrical engineering from KAIST (Korea Advanced Institute of Science and Technology), Korea in 1975 and the Doctor degree in electric and information from ENSEEIHT, France in 1979. He joined the faculty of Korea University in 1982, where he is currently a Professor of Electronic Engineering. Prior to joining Korea University, he was Assistant Professor of Electronic Engineering in Ajou University, Suwon, Korea. He was with NIST(National Institute of Standards and Technology) in U.S.A., as a visiting scientist in 1991.His research interests include the distributed system, communication networks and protocols, information network, intelligent network and multimedia communication system.

Dr.An was an advisory committee of ETRI

(Electronics and Telecommunications Research Institute) and Chairman of IEEE Seoul Section. $\mathrm{He}$ is a member of the ACM and IEEE. 